\title{
Study of DSSSD detector response in the inter-strip region using a proton micro-beam
}

\author{
Laura Grassi ${ }^{1, a}$, Domenico Torresi², Luis Acosta ${ }^{2}$, Pierpaolo Figuera ${ }^{2}$, Maria Fisichella ${ }^{2,3}$, \\ Veljko Grilj ${ }^{1}$, Milko Jakšić ${ }^{1}$, Marcello Lattuada ${ }^{2,4}$, Tea Mijatovićc ${ }^{1}$, Matko Milin ${ }^{5}$, Lovro Prepolec ${ }^{1}$, \\ Natko Skukan ${ }^{1}$, Neven Soić ${ }^{1}$, Vedrana Tokić ${ }^{1}$ and Milivoj Uroić ${ }^{1}$ \\ ${ }^{1}$ Ruđer Bošković Institute, HR-10000, Zagreb, Croatia \\ 2 INFN, Laboratori Nazionali del Sud, I-95125, Catania, Italy \\ ${ }^{3}$ Dipartimento di Fisica, Università di Messina, I-98166, Messina, Italy \\ ${ }^{4}$ Dipartimento di Fisica e Astronomia, Università di Catania, I-95125, Catania, Italy \\ ${ }^{5}$ Department of Physics, University of Zagreb, HR-10000, Zagreb, Croatia
}

\begin{abstract}
Double Sided Silicon Strip Detectors (DSSSDs) are segmented silicon detectors widely used for the detection of charged particles. When a particle hits the inter-strip gap between two adjacent strips, energy measurement can be affected by different effects reducing the efficiency of output signal generation. With the purpose to study this behaviour, an experiment was performed at the scanning proton micro-beam facility of the Ruđer Bošković Institute (RBI). Response of two detectors of thickness 75 and $998 \mu \mathrm{m}$ is examined as a function of the beam impact position and bias voltage. Results show that the effective width of the inter-strip gap, which is linked to the efficiency for full energy detection, depends on the experimental conditions and detector settings.
\end{abstract}

\section{Introduction}

Double Sided Silicon Strip Detector (DSSSD) is a silicon detector with both surfaces segmented into 16 mutually orthogonal strips. The segmentation is made of $\mathrm{SiO}_{2}$, an insulator performing electrical isolation between adjacent strips. When a charged particle hits the active area of the detector, its response gives information on deposited energy and 2D impact position of the particle. Change of the segmented silicon detector response due to modifications of the charge collection in the proximity of the $\mathrm{SiO}_{2}$ inter-strip gap was examined in existing literature [1-6].

Regular detector response corresponds to those events when only one strip on the front side and one strip on the back side produce signals with the same amplitudes (till the own resolution). Particles hitting the region close to the inter-strip gap, could be effected by signals with low energy amplitutde. Such events are usually neglected in analysis of data from nuclear experiments and the detection efficiency is assumed constant and uniform over the detector active area. The results of measurements reported in this paper show that the detector region from where anomalous response originates, is sensitive to the detector settings and characteristics like bias voltage and detector thickness. Moreover, the size of this region doesn't correspond to geometrical size of the $\mathrm{SiO}_{2}$ gap between the active strips.

${ }^{\mathrm{a} C}$ Corresponding author: laura.grassi@irb.hr 

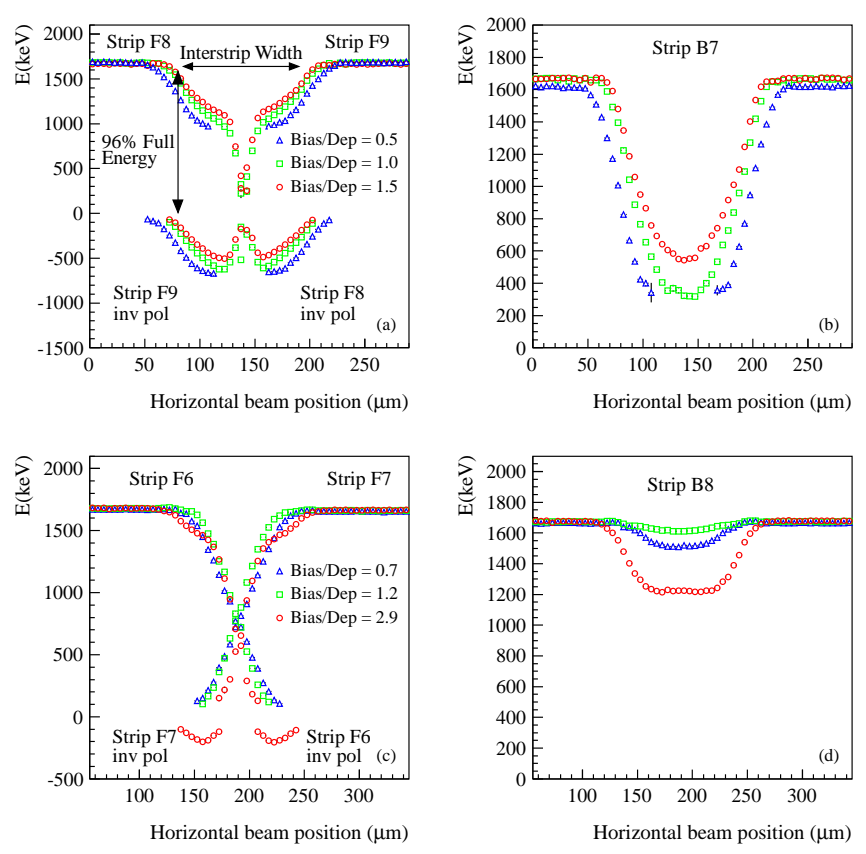

Figure 1. Data taken with the proton micro-beam of $1700 \mathrm{keV}$ energy for three different bias voltages. (a), (b) $998 \mu \mathrm{m}$ thick DSSSD: micro-beam scanned a rectangular shape on the detector including parts of strips 8 and 9 on the front side, $\mathrm{SiO}_{2}$ zone between them and strip 7 on back side. (a) energy response of the front strips against the horizontal beam position for three different bias settings; (b) back strip energy response against the horizontal beam position. (c), (d) DSSSD $75 \mu \mathrm{m}$ thick: scanned area includes parts of the front side strips 6 and 7, $\mathrm{SiO}_{2}$ zone between them and back side strip 8. (c) energy response of the front strips against the horizontal beam position for three different bias settings; (d) corresponding back strip energy response against the horizontal beam position.

\section{Experimental set up}

The examined DSSSDs are model W1 manufactured by Micron Semiconductor Ltd with thickness of 998 and $75 \mu \mathrm{m}$. Their active area is $50 \times 50 \mathrm{~mm}^{2}$ and each side is divided into 16 parallel strips, $3 \mathrm{~mm}$ wide. The junction (front) side is p-type silicon and the ohmic (back) side is n-type. The strips on the front side are perpendicular to the ones on the back side, giving a granularity of $16 \times 16=256$ square shaped pixels. Therefore the detector position resolution is $3 \times 3 \mathrm{~mm}^{2}$. The $100 \mu \mathrm{m}$ wide $\mathrm{SiO}_{2}$ layer is an insulator that separates strips electrically. The metallization is made of a $3000 \AA$ thick aluminum layer, and the silicon dead layer of thickness $\backsim 0.5 \mu \mathrm{m}$ is also present. The full depletion voltage declared by the manufacturer for the thick and thin detector are $180 \mathrm{~V}$ and $3 \mathrm{~V}$, respectively. The response of the two DSSSDs was studied by using the proton micro-beam at beam energy of $1700 \mathrm{keV}$ (low intensity $\sim \mathrm{fA}$ ), and different bias voltage operating conditions: under-depleted, fullydepleted and over-depleted.

In this experiment the response of two adjacent strips was studied in two configurations labeled $\mathrm{A}$ and $\mathrm{B}$. Configuration A (B) corresponds to the micro-beam focused the $\mathrm{SiO}_{2}$ layer on the front (back) side. Micro-beam system [7] provides the proton beam spot on the detector of $1 \mu \mathrm{m}$ size which can be 
moved in both directions inside rectangular shaped scanning area. The horizontal and vertical position of the beam is recorded together with the energy response of all 32 strips of the DSSSD. With the aim to record inverted polarity signals from the strips, MESYTEC MPR 16 pre-amplifier output of each strip was split into two electronics branches (SILENA 716 programmable amplifiers) set to analyse signals of normal and inverted polarity, giving at the end 64 energy signals in data acquistion system.

\section{Results}

With the aim to compare the various measurements, results are presented as averaged values of position and energy measured for each recorded event. Data are sorted in position bins of $5 \mu \mathrm{m}$ chosen to optimize position resolution of the micro-beam system with beam spot size of $\sim 1 \mu \mathrm{m}$ and step of $\sim 4 \mu \mathrm{m}$. Estimated position error is $\pm 2.5 \mu \mathrm{m}$. For each position bin the mean value of energy and its standard error were calculated from recorded data.

The proton micro-beams of $1700 \mathrm{keV}$ incident energy and few pps intensity were focused directly on the surface of the detector in the region around the $\mathrm{SiO}_{2}$ zone. Figure 1 shows averaged data of the measurements with detectors in configuration A and for three bias settings. Each panel shows the energy response of the front and back side strips against the horizontal beam position, (a) and (b) for the thick detector, (c) and (d) for the thin one. When protons hit area of the strips far from the $\mathrm{SiO}_{2}$ zone, full induced signal is recorded for one strip on the front and back side. If the micro-beam is focused close to the $\mathrm{SiO}_{2}$ zone of the front side, the energy signal amplitude of normal polarity from one strip decreases, and at the same time opposite polarity signal appears in the adjacent strip on the front side (see figure 1 (a) and (c)). When the beam moves towards the central position of the $\mathrm{SiO}_{2}$ zone, the opposite polarity signal amplitude first increases reaching its maximum value, and then it decreases. In the real center of the interstrip gap there are two normal polarity pulses from the front side but with reduced energy amplitude.

Results show that the behavior of the two detectors for proton position in proximity of the $\mathrm{SiO}_{2}$ zone is quite different against increase of the bias voltage. Energy response of the thick detector improves for both the front and back side strips, due to the decreasing of inverted polarity pulse amplitudes (red empty circles in figure 1 (a) and (b) panels). In the case of thin detector, inverted polarity pulses appear only when the applied bias is much larger than the depletion voltage.

With the aim to examine the dependence of this region to the detector settings, we define inter-strip gap as the difference between the two beam positions where the energy signals from the two adjacent strips drop below $96 \%$ of their full amplitude. The threshold of $96 \%$ is arbitrarily chosen to compare the different shapes of all averaged plots.

The figure 2 (a) shows the inter-strip widths of two detectors for measurements in configurations A (full markers) and B (empty markers) against the ratio between the bias applied to full depletion voltage declared. The thick detector is characterized by an overall larger inter-strip gap value than the thin DSSSD.

The inter-strip widths corresponding to the configuration A are closer to the nominal width of the $\mathrm{SiO}_{2}$ zone than the values obtained for the measurements in configuration $\mathrm{B}$. In this latter case, inter-strip gap of the back side of the thick detector is significantly larger than the one of the front side (empty triangles in figure 2(a)). Contrariwise, the inter-strip width of the back side of the thin detector is smaller than the one of the front side (empty circles in figure 2(a)). Possible explanation is that at this beam energy, protons are stopped at distance of about $35 \mu \mathrm{m}$ from the front surface, corresponding to a different charge collection effects due to the different thickness of the detectors.

In both detectors, only when protons hit the inter-strip region of the front side (configuration A), the back strip energy response is characterized by a reduced signal amplitude (see figure 1 (b) and 

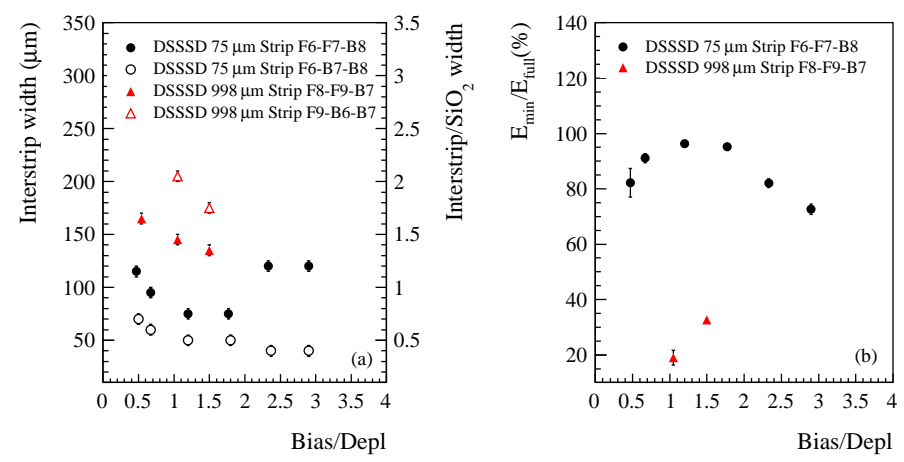

Figure 2. (a) Results show the dependence of the inter-strip width on the ratio bias/depletion voltage for the two DSSSDs. Right axis shows the ratio between the measured inter-strip gap and the $\mathrm{SiO}_{2}$ width. The names F0 to F15 to indicate the 16 front strips and B0 to B15 to indicate the 16 back strips. Full and empy markers corrispond to the beam focused in the inter-strip gap of the front side and the back side, respectively. (b) Plot presents dependence of ratio $E_{\min } / E_{\text {full }}(\%)$ on ratio bias/depletion voltages for both detectors set on configuration A.

(d)). With the aim to quantify this effect, we consider the ratio between the minimum energy $\left(E_{\min }\right)$, and the full energy far from the $\mathrm{SiO}_{2}$ zone $\left(E_{f u l l}\right)$ where no inverted polarity pulse or charge sharing occurs. The minimum energy value for the thick detector corrisponds to the vertex of a parabolic fit of the average plot in figure 1 (b). In the case of the thin detector, the energy response against the beam position plots show a saturation plateau (figure $1(\mathrm{~d}))$. The minimum value $\left(E_{\min }\right)$ of the energy was found using the mean value of this plateau. The results for both detectors are presented in figure 2 (b). For the thin detector, the best work condition corresponds to bias close to the depletion voltage, leading to smaller values of inter-strip gap and to energy amplitudes $\backsim 100 \%$. Behaviour of the thick detector is very different: the ratio $E_{\min } / E_{\text {full }}(\%)$ increases for higher bias, but for measured detector settings it is far below the full energy amplitude.

In conclusion, the results indicate that the front and back effective inter-strip width, which is related to the DSSSD efficiency for full deposited energy detection, depends on the detector thickness and on the operating conditions. Therefore complete characterization of the used DSSSDs is required.

Further measurements and simulations of the detector response are in progress.

\section{References}

[1] J. Yorkston and A. C. Shotter, Nucl. Instr. and Meth. A 262, 353-358 (1987)

[2] Y. Blumenfeld et al., Nucl. Instr. and Meth. A 421, 471-491 (1999)

[3] V. Eremin et al., Nucl. Instr. and Meth. A 500, 121-132 (2003)

[4] S. Takeda et al., Nucl. Instr. and Meth. A 579, 859-865 (2007)

[5] T. Poehlsen et al., Nucl. Instr. and Meth. A 700, 22-39 (2013)

[6] D. Torresi, Nucl. Instr. and Meth. A 713, 11-18 (2013)

[7] M. Jakšić et al., Nucl. Instr. and Meth. B 260, 114-119 (2007) 